\title{
An Efficient Method for Detection and Classification of Pulmonary Neoplasm based on Deep Learning Technique
}

\author{
${ }^{* 1}$ C.Venkatesh, ${ }^{2}$ L.Siva Yamini \\ ${ }^{1,2}$ Assistant Professor, Electronics and Communication Engineering, AITS Rajampet, A.P, India \\ *1venky.cc@gmail.com \\ ${ }^{2}$ sivayamini470@gmail.com
}

Received: $13^{\text {th }}$ November 2020, Accepted: 25 th January 2021, Published: $26^{\text {th }}$ February 2021

\begin{abstract}
Cancers of the lung and pancreas are two of the most frequent cancers. While lung cancer is the leading cause of cancer-related deaths worldwide, pancreatic cancer has the worst prognosis, with only a $7 \% 5$-year survival rate in the US. In lung and pancreatic cancer screening, accurate nodule detection is critical. Typically, radiologists discover these cancers on medical pictures through a thorough review of CT images, which takes a long time and is inexact. As a result, an unique hybrid model for accurately detecting such malignancies is offered. Because it is a progressive diagnostic tool for medical reasons, the proposed method is accomplished using image processing techniques. There are two stages to the proposed technique. In the first stage, both supervised and unsupervised deep learning algorithms and SVM techniques are used to classify whether lung and pancreatic tumors are malignant or benign, as well as to improve tumor characterization. The tumor component is discovered in the second stage utilizing Convolution Neural Networks and Transfer Learning. The CT image is first pre-processed from the data base for scaling and noise removal. After that, the image is categorized using supervised and unsupervised learning techniques. CNN segments the tumor part if the tumor in the image is assessed as malignant. Finally, several metrics such as accuracy, specificity, and sensitivity are calculated and compared to previous method findings.
\end{abstract}

\section{Keywords: Deep learning techniques, Convolution Neural Network, Neoplasm, Lung, Pancreas}

\section{Introduction}

Our research makes advantage of the deep learning idea, which is becoming increasingly popular as technology advances. We propose the concept of deep learning as a means of reducing human labour. In the early stages, it's difficult to identify because of the lack of particular (or missing) symptoms, which are aggravated by the overlap of COPD symptoms (COPD). An accurate assessment of lung nodules' progression is necessary for an early CT diagnosis. Inter observer and scan variability make it difficult to do a manual radiological evaluation. A new and promising area of medical image analysis, deep learning architectures, will be used in this project to develop novel solutions to tackle some of the most pressing medical imaging challenges, such as early detection of lung cancer in patients with nodules found on CT scans, co-existing emphysema, concurrent infection, varying levels of aspiratory effort, and variable acquisition parameters.

As the second most common cancer in both men and women worldwide, lung cancer also has the greatest death rate. This is due to the fact that it often goes unnoticed for long periods of time, resulting in poor treatment outcomes when it is finally discovered. Despite this, the United Kingdom does not have a programme for early identification of lung cancer. Inadequate diagnostic tests, especially imaging, and exorbitant expenses make this unaffordable for a huge segment of the population. Lung cancer screening is now limited to people who have been identified as having a high risk of getting lung cancer, such as smokers or COPD patients (or both), but this research is hoped to serve as solid foundation for future national screening programme.

Radical imaging is seeing a rise in the use of machine learning methods. Unsupervised and semi-supervised algorithms, particularly those using deep learning architectures, have the potential to overcome the problem of a lack of tagged image data. Lung CT screening trials using off-the-shelf convolution neural networks (CNNs) for nodule classification and detecting respiratory illness from chest radiographs using CNNs with a first layer trained on natural pictures are two of the most pressing areas of current lung imaging research. For follow-up 
imaging or for establishing spatial correlation, none of these trials were conducted. A 2-layer CNN and deep feature learning were used in to solve the issue of MR brain spatial normalization. To represent a set of observed image patches, they employ an unsupervised deep learning approach that trains basis filters directly. The coefficients of these filters can then be used to detect correspondence during image registration.

This study is influenced by examining the use of diverse forms of data for deep feature learning (including natural images and also high-contrast lung CT scans), as well as by incorporating learned features into a deformable registration framework. A Markov random field (MRF)-driven registration system can incorporate both high- and low-contrast hierarchical image properties and complex motion patterns that we shall discover. Lung nodules in patients at risk of cancer can be detected and tracked by serial registration of low-dose, lowcontrast lung CT scans.

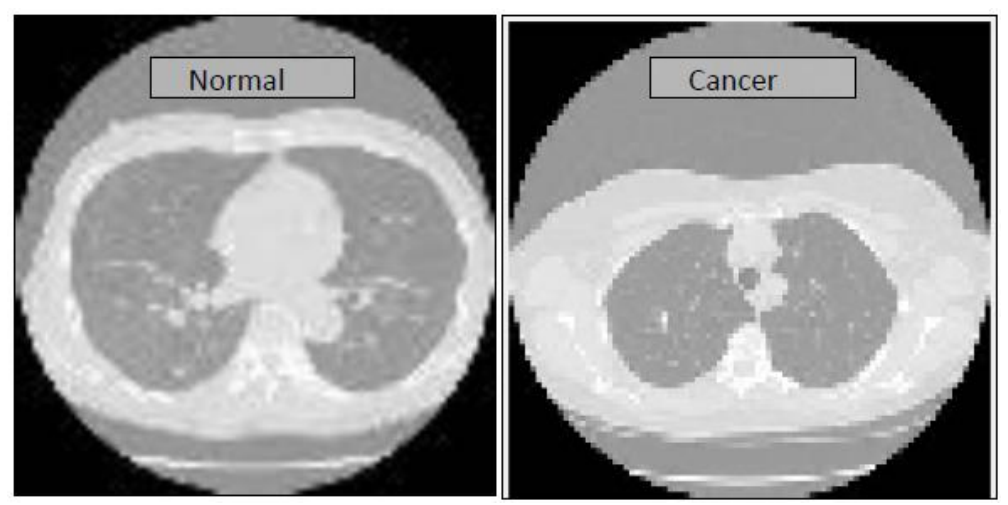

Fig 1: Typical normal and cancerous CT Scans

\section{Review of Literature}

It has been traditional to use nodule segmentation, computation and selection of low-level image features and the use of a classifier/regress or an approach by which different physical statistics such as intensity measures were extracted, and class labels were obtained using Artificial Neural Networks for stratification (classification) of lung nodules' risk. Spherical harmonics were used to identify the geometry of lung nodules that had been segregated using appearance-based models. Classification was done using k-nearest neighbors in the final phase. Another technique involved converting 2D texture elements such as Local Binary Patterns, Gabor, and Heraldic to 3D.. Local Binary Patterns, Gabor, and Heraldic 3D texture elements were employed in a second approach that was previously only available in 2D. The last step was to use a Support Vector Machine to classify the data (SVM). Using 3D active contours, Way et al. segmented nodules and then used the rubber band straightening procedure to straighten them.

There were certain shortcomings in the existing approaches, which are listed below:

Nodule segmentation, computation, and selection of low-level parameters from the image, as well as the application of a classifier/regressor, have typically been required for lung nodule risk categorization. By employing Artificial Neural Networks, a variety of physical statistics, including intensity measures, were collected and class labels were generated. Spherical harmonics were used to identify the geometry of lung nodules that had been segregated using appearance-based models.

Finally, the k-nearest neighbors classification was used to determine the final groupings. Local Binary Patterns, Gabor, and Heraldic 3D texture elements were employed in a second approach that was previously only available in 2D. To make up for the flaws in the original approach, we've developed a convolutional neural network.

This method for detecting lung and pancreatic tumors comprises two steps, which we have incorporated into it. In the first stage, a CT picture was used as an input. Then it undergoes a classification procedure to see if there is a tumour. Procedures are stopped if there isn't a tumour. Using the U-net Architecture, if the tumour is available, it is segmented to create individual parts. 
SVM: An SVM model is essentially a hyperplane representation of discrete classes in multidimensional space. In order to reduce error, SVM will iteratively construct the hyper plane. Datasets are divided into classes by SVM in order to find the maximum marginal hyperplane (MMH).

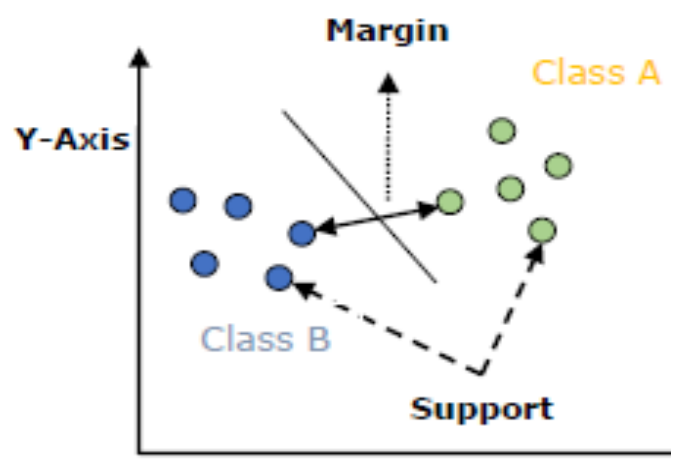

Fig 2: Classification of SVM

- Support Vectors: Data points that are closest to the hyper plane are called support vectors. Separating line will be defined with the help of these data points.

- Hyper plane: As we can see in the above diagram, it is a decision plane or space which is divided between a set of objects having different classes.

- Margin: It may be defined as the gap between two lines on the closet data points of different classes. It can be calculated as the perpendicular distance from the line to the support vectors. Large margin is considered as a good margin and small margin is considered as a bad margin.

\section{Research Methodology}

For the development of lung cancer diagnoses, this study summarizes recent developments in machine learning. Neural networks that are not labelled are known as deep neural networks (DNNs). In the majority of cases, it's employed for analyzing visual images. CNN, compared to other image classification techniques, requires very minimal preprocessing. This approach takes a long time to compute because to the enormous amount of photographs that is entered. Comparatively, it exhibits a delay and lower precision than PNN.

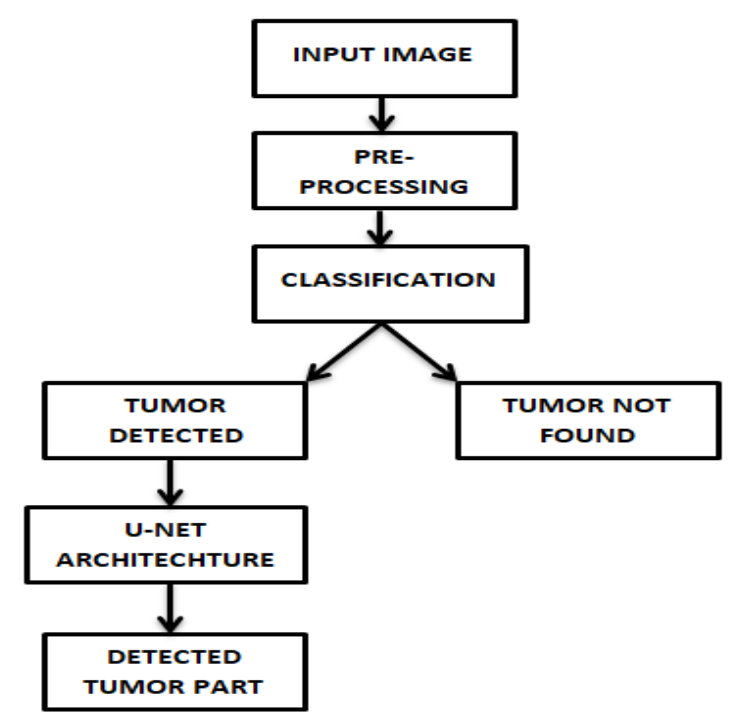

Fig 3: Block diagram of proposed method 
Convolution neural network: Artificial Intelligence has made significant progress in closing the gap between human and computer capabilities. Researchers and hobbyists alike work on a variety of facets in the field to achieve incredible results. The field of computer vision is one of several such disciplines.

The goal of this field is to enable machines to see and perceive the world in the same way that humans do, and to use that knowledge for a variety of tasks such as image and video recognition, image analysis and classification, media recreation, recommendation systems, natural language processing, and so on. Advancements in Computer Vision using Deep Learning have been built and developed over time, mostly through the use of a single algorithm, the Convolution Neural Network.

Preprocessing, feature extraction using CNN, feature selection using offline genetic algorithm training, and classification are the four processes in the proposed system for nodule identification. The output is either normal or malignant.

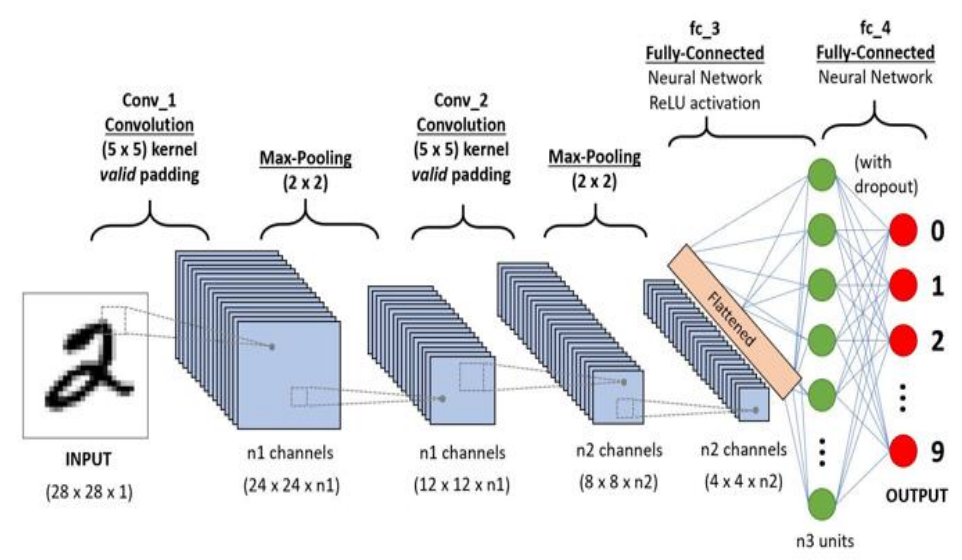

Fig 4: A CNN sequence to classify handwritten digits

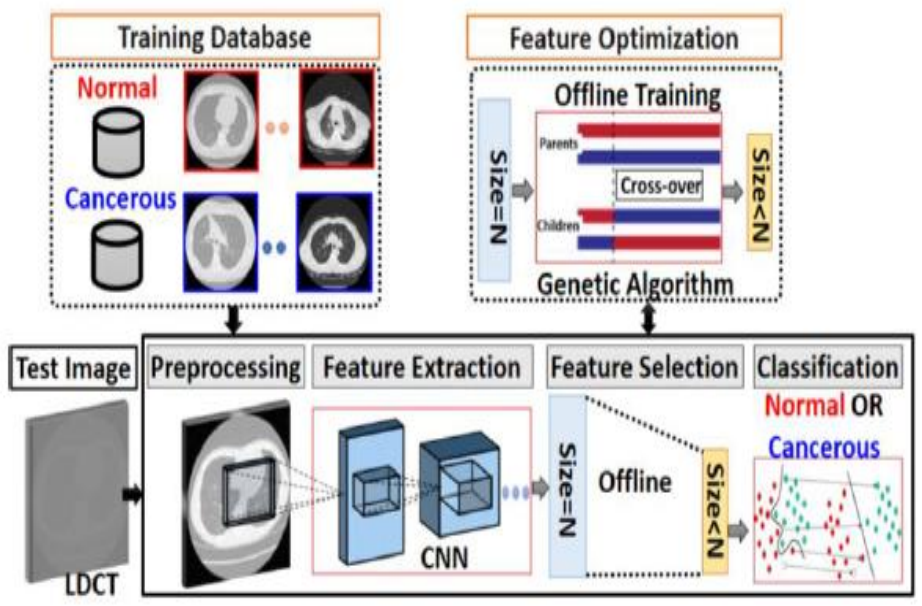

Fig 5: Processes in the proposed system for nodule identification

\section{Pre - processing}

Although the most relevant input data has been chosen, it must be pre-processed, or the neural network will not generate correct results. This decreases the quantity of inputs to the network, making it easier for it to learn. It eliminates undesired signals from CT scans. Color photos are converted to grey-level coding.

As seen in Figure 6, the preprocessing procedure is broken down into three parts. The raw image's contrast is first improved using the histogram stretching approach. Second, to remove scanner noise, a smoothing Wiener filter is applied. Finally, the image is cropped to fit the CNN model's typical size for feature extraction.

Raw data is preprocessed in three steps: histogram stretching to boost image contrast, image smoothing with the Wiener filer, and cropping to the CNN model's typical input size for feature extraction. 


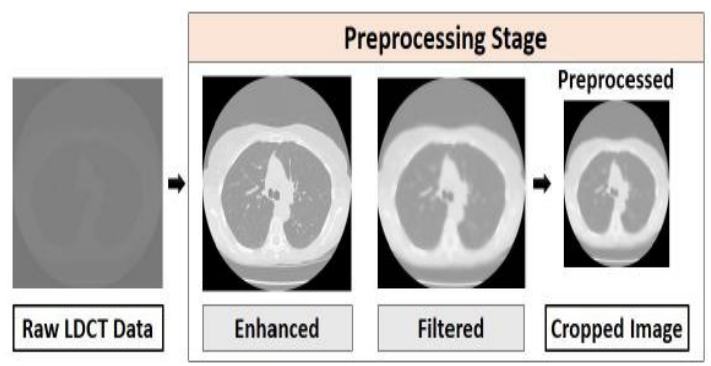

Fig 6: Preprocessing of Raw data

\section{Classification}

U-Net is a Convolution Neural Networks (CNN)-based method. In the identified output image, this is used for tumor segmentation. The remaining half of the training photos is used to train the classifier.

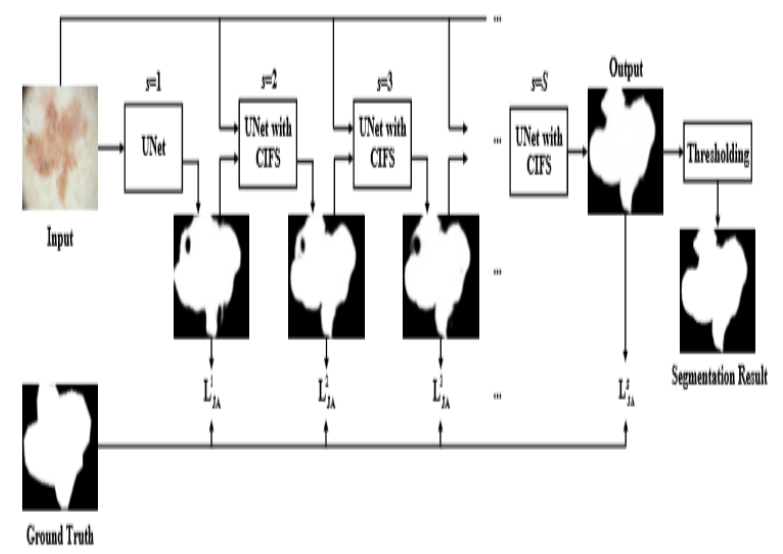

Fig 7: U-Net architecture

The technique of segmentation divides an image into areas. It is a method of image processing that allows us to distinguish between objects and textures in photographs. It plays a key function in tumor detection and remote sensing

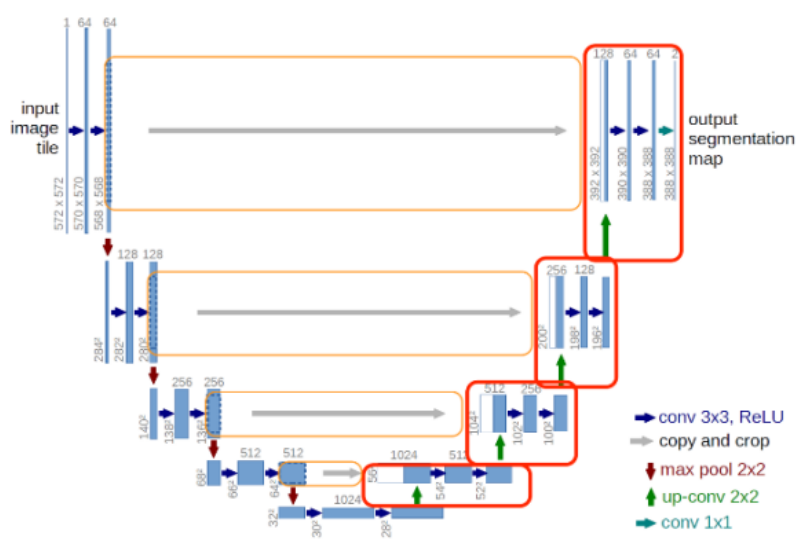

Fig 8: U-Net models

U-Net gets its name from the architecture, which resembles the letter $\mathrm{U}$ when viewed, as illustrated in the diagram above. A segmented output map is created from the input photos. In the second half, the most unique characteristic of the architecture there is no fully connected layer in the network. The convolution Layers are the only ones that are used. A ReLU activation function is used to start each normal convolution operation. The segmented image is delivered as the final output after the operation is completed. 


\section{Results}

Table 1: Comparative parameters

\begin{tabular}{|l|l|l|}
\hline PARAMETERS & EXISTING & PROPOSED \\
\hline ACCURACY & 94.8625 & 99.7989 \\
\hline SPECIFICITY & 92.514 & 99.4218 \\
\hline SENSTIVITY & 85.625 & 100 \\
\hline $\begin{array}{l}\text { DICE } \\
\text { COEFFICIENT }\end{array}$ & 0.8875 & 0.9971 \\
\hline $\begin{array}{l}\text { JACCARD } \\
\text { COEFICIENT }\end{array}$ & 0.8754 & 0.9942 \\
\hline
\end{tabular}

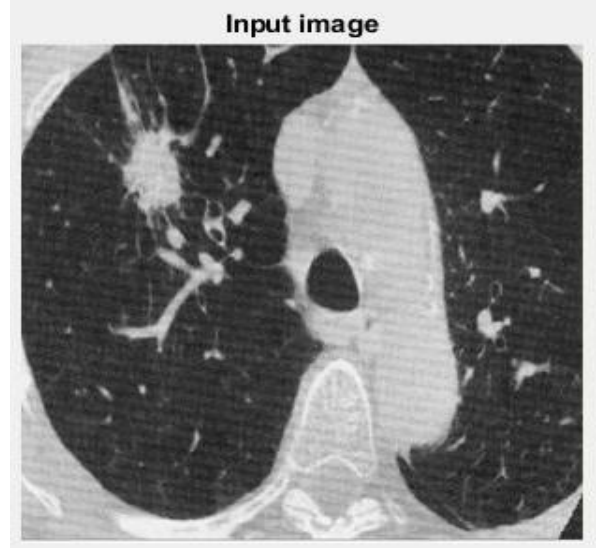

Fig 9: Input image of Lung

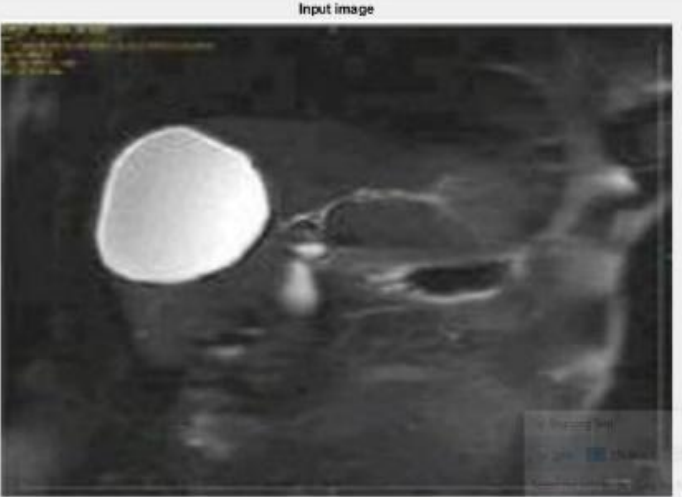

Fig 10: Input image of Pancreas

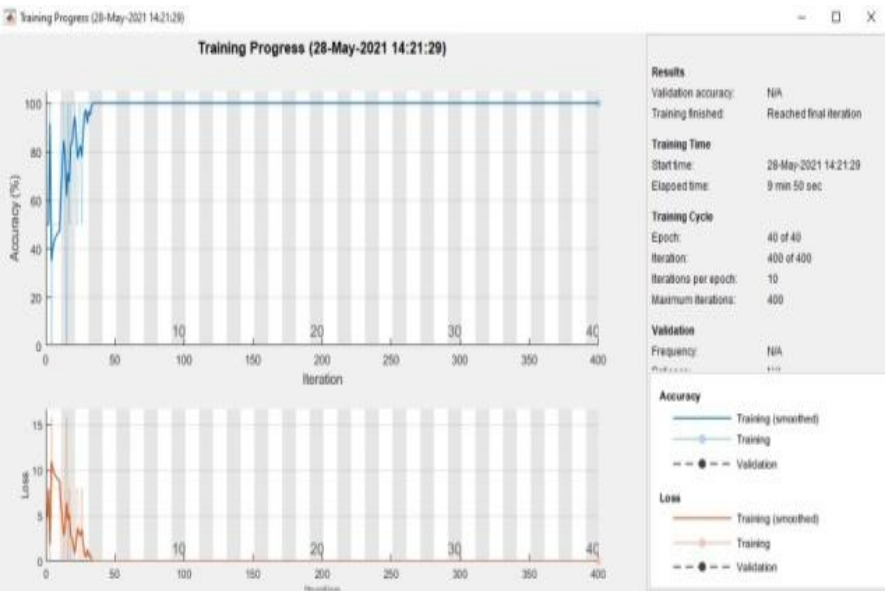

Fig: 11 Training Process for Lung

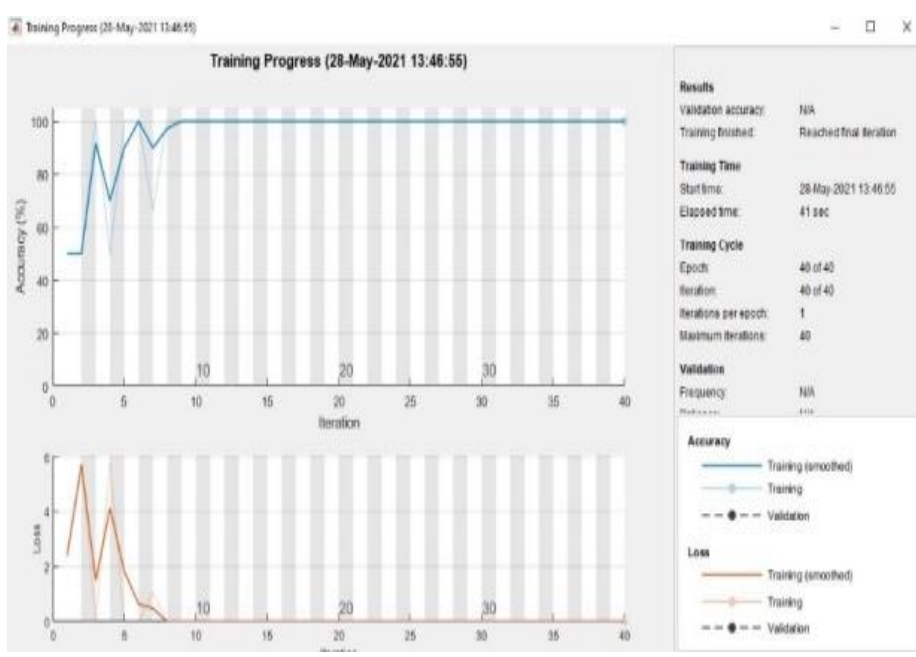

Fig12 Training Process for Pancreas 


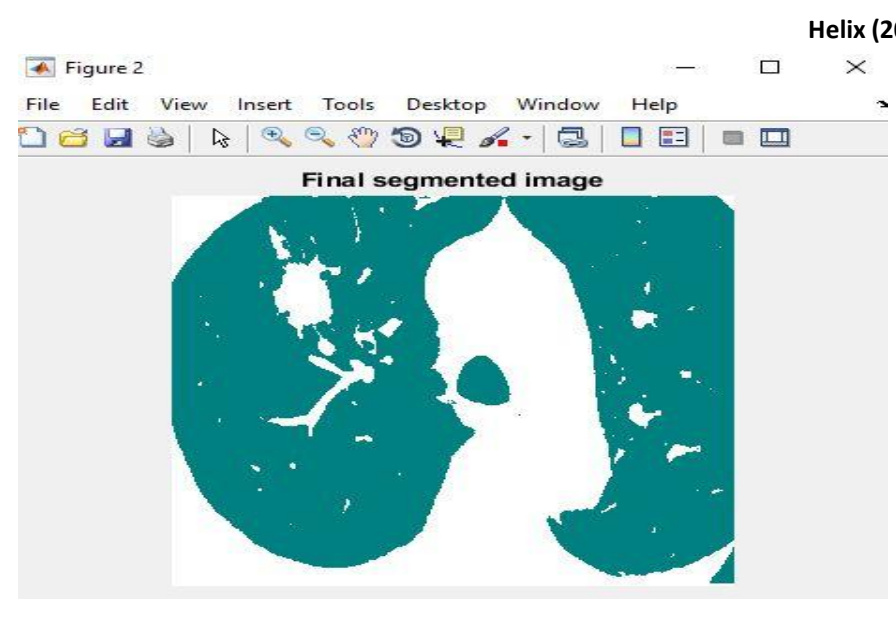

Fig 13: Segmented Output Lungs

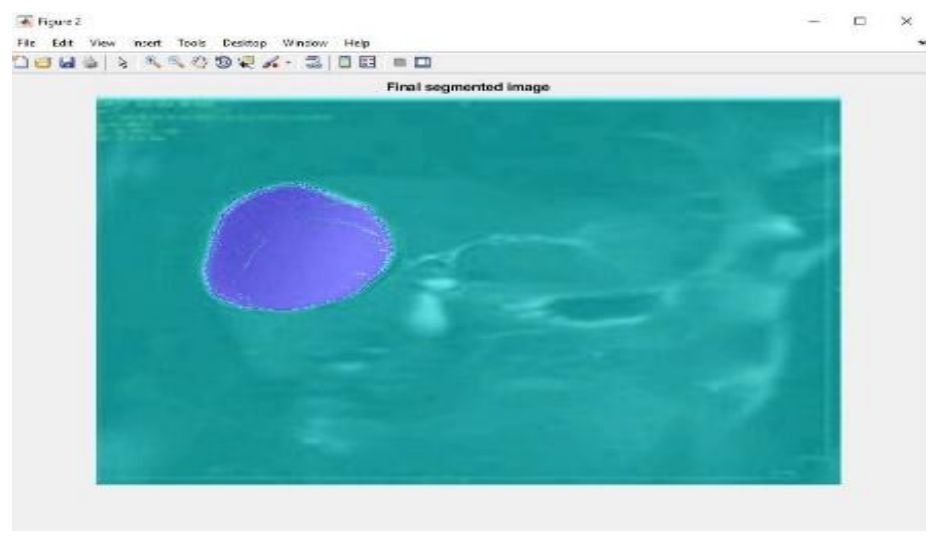

Fig 14: Segmented Output for Pancreas

\section{Conclusion}

Lung cancer is one of the world's most hazardous diseases. With current segmentation approaches, detecting cancer at an early stage is fairly challenging. Lung cancer can be diagnosed correctly and detected early, increasing the chances of survival. Lung cancer, on the other hand, is diagnosed by radiologists, which takes much longer and is less accurate. As a result, a U-net design based on image processing is proposed for accurate detection. First, CT scan images are obtained from a database in this project. To extract information from the scan images without noise, $\mathrm{CNN}$ is used to process them. The suggested Convolution Neural Network accurately classifies the region of interest, i.e., tumor or cancer, in the filtered images (CNN) The tumor component is discovered during the segmentation phase utilizing U-Net Architecture. Different parameters such as specificity, sensitivity, and accuracy were also calculated.

\section{References}

1. A. Esteva, et al., "Dermatologist-level classification of skin cancer with deep neural networks," Nature, vol. 542, no. 7639, pp. 115-118, 2017

2. El-Baz, A., Nitzken, M., Khalifa, F., Elnakib, A., Gimelfarb, G., Falk, R., El-Ghar, M.A.: 3D shape analysis for early diagnosis of malignant lung nodules. In: IPMI. pp. 772-783. Springer (2011)

3. Han, F., Wang, H., Zhang, G., Han, H., Song, B., Li, L., Moore, W., Lu, H., Zhao, H., Liang, Z.: Texture feature analysis for computer-aided diagnosis on pulmonary nodules. Journal of Digital Imaging 28(1), 99- 115 (2015)

4. J. G. Elmore, et al., "Pathologists' diagnosis of invasive melanoma and melanocytic proliferations: observer accuracy and reproducibility study." British Medical Journal, vol. 357, pp. j2813, 2017.

5. J. Long, et al., "Fully convolutional networks for semantic segmentation," IEEE Transactions on Pattern Analysis and Machine Intelligence, vol. 39, no. 4, pp. 640-651, 2017

6. L. Bi, et al., "Dermoscopic image segmentation via multi-stage fully convolutional networks," IEEE Transactions on Biomedical Engineering, vol. 64, no. 9, pp. 2065-2074, 2017

7. Lee, M., Boroczky, L., Sungur-Stasik, K., Cann, A., Borczuk, A., Kawut, S., Powell, C.: Computeraided diagnosis of pulmonary nodules using a two-step approach for feature selection and classifier ensemble construction. Artificial Intelligence in Medicine 50(1), 43-53 (2010)

8. R. L. Siegel, et al., "Cancer statistics, 2018," CA: A Cancer Journal for Clinicians, vol. 68, no. 1, pp. 730,2018

9. S. Pathan, et al., "Techniques and algorithms for computer aided diagnosis of pigmented skin lesions-a review," Biomedical Signal Processing and Control, vol. 39, no. 2018, pp. 237-262, 2017

10. Sadot, E., Basturk, O., Klimstra, D.S., Gonen, M., Anna, L., Do, R.K.G., “' DAngelica, M.I., DeMatteo, R.P., Kingham, T.P., Jarnagin, W.R., et al.: Tumor-associated neutrophils and malignant progression in intraductal papillary mucinous neoplasms: an opportunity for identification of highrisk disease. Annals of surgery 262(6), $1102(2015)$

11. Way, T.W., Hadjiiski, L.M., Sahiner, B., Chan, H.P., Cascade, P.N., Kazerooni, E.A., Bogot, N., Zhou, C.: Computer-aided diagnosis of pulmonary nodules on CT scans: segmentation and classification using 3D active contours. Medical Physics 33(7), 2323-2337 (2006)

12. Y. Yuan, et al., "Automatic skin lesion segmentation using deep fully convolutional networks with jaccard distance,” IEEE Transactions on Medical Imaging, vol. 36, no. 9, pp. 1876-1886, 2017 